\title{
Building a Digital Platform for Behavioral Intervention Technology Research and Deployment
}

\author{
Jim Warren \\ University of Auckland \\ jim@cs.auckland.ac.nz
}

Sarah Hetrick

University of Auckland

s.hetrick@auckland.ac.nz

\author{
Sarah Hopkins \\ University of Auckland \\ s.hopkins@auckland.ac.nz
}

\author{
Andy Leung \\ University of Auckland \\ andy.leung@auckland.ac.nz
}

\author{
Sally Merry \\ University of Auckland \\ s.merry@auckland.ac.nz
}

\begin{abstract}
While there is demonstrated potential for behavioral intervention technologies (BITs) to improve access to beneficial mental health interventions, there is a continuing need for research to evaluate effectiveness of new and existing applications for specific population groups. This paper reports experience in developing a digital platform to support delivery of an ecosystem of online mental health screening and behavioral intervention technologies to promote the wellbeing of New Zealand youth. Key platform requirements center on identity management, usage and assessment tracking, and implementation of research protocol workflow. A reusable and scalable solution based on Web services is presented in the context of ongoing iterative development and end user studies. The platform is providing consistent research and service delivery support for multiple concurrent threads of the overall youth mental health program.
\end{abstract}

\section{Introduction}

The potential of electronic interventions to improve mental health for young people, especially groups at higher risk, is promising and in urgent need of being realized. Depressive and anxiety disorders along with self-harm including suicide are three of the five leading causes of loss of disability-adjusted life years for the 15-19 age group globally [1]. As compared to the New Zealand (NZ) European population, Māori (indigenous New Zealanders) have a disproportionately higher burden of mental health [2]. Providing e-Health interventions to support and maintain mental health and wellbeing has been shown to lead to improved emotional well-being for youth in general [3] and in school settings [4]. This demonstrated effectiveness, along with potential accessibility and scalability of e-Health interventions, suggests its application to address mental health needs including specific inequalities.

Despite evidence for effectiveness of e-Health interventions for youth mental health, there is ongoing need for research studies in this space. High non-adherence (failure to fully experience the intervention as intended) is the norm for electronic mental health interventions in open access; and while adherence is better in clinical trials, rates of nonadherence and drop-out are still non-trivial [5]. Elements of persuasive system design have been enumerated, including primary task, dialogue, system credibility and social support [6]. These provide a guide to developers of new e-Health apps. Further, the inclusion of dialog support elements (e.g. praise, rewards, reminders) has been shown to be associated with better adherence to Web-based interventions [7].

For our work, we must address the needs of Māori and Pasifika youth and (with considerable overlap) NZ young people in areas of high socio-economic deprivation, fitting designs to their cultures, preferences and contexts of use. Moreover, changing trends in technology mean that a proven intervention from a decade ago may not be in the right format today. As such, we require nearly-continuous studies of usability, use and health benefits of new, updated and repurposed apps. Unfortunately, this need for research incurs a need for continuous research funding to support administering clinical trials and monitoring uptake and use of deployed interventions.

In this paper we report on our experience in the context of a program of applied research for promoting the wellbeing of NZ youth through a suite of e-Therapy apps. To support our work a digital 
platform has been developed that implements key functions for research into and ongoing delivery of mental health promoting applications. The objective was to provide efficiency, quality, maintainability and scalability of the applied research program by developing a re-usable and tailorable solution (a 'digital platform') addressing recurring requirements. In the next section we describe the research context and the methodology of the digital platform design and development effort, including its relationship to the broader program of work. We then present findings from phases of the research ranging from initial requirements analysis, through proof of concept prototyping and wider application, and on to our ongoing work to support the workflow of protocols of field trials and deployments. We conclude with discussion of outstanding issues and opportunities for digital platforms in e-Therapy.

\section{Research context and methodology}

Initial motivation for the present work came from the success of SPARX (Smart, Positive, Active, Realistic, X-factor thoughts). SPARX provides cognitive behavioral therapy (CBT) for youth in a gamified adventure format with seven modules to be completed over a $4-7$ week period. It has been shown to be no less effective than usual treatment (face-toface counseling delivered by trained counselors and clinical psychologists) [8]. It is available for public use in NZ as a Web application and in updated format as a mobile app (https://www.sparx.org.nz/). Our e-Health intervention concept was further informed by the electronic Case-finding and Health Assessment Tool (eCHAT), a Web-based self-report screening tool designed to assess risky health-related behaviors and mental health concerns, with a help question on each area (e.g. depression, anxiety, alcohol use, gambling) so the user can prioritize areas they want help with [9]. Working with NZ youth, eCHAT has been tailored for adolescents as YouthCHAT, including addition of sexual health questions and modification of the alcohol and drug modules from the general/adult version [10].

From 2016, a program of applied research called HABITs (Health Advances through Behavioural Intervention Technologies) has been funded through an NZ National Science Challenge for promotion of youth health, "A Better Start - E Tipu e Rea." The aim of HABITs is to deliver e-Health interventions promoting the wellbeing of NZ teens. HABITs leverages the experience from SPARX and YouthCHAT. The initial plan was to utilize: (1) e-Screening - improved detection of youth mental health problems through YouthCHAT; and (2)
e-Therapy - delivery of Behavioral Intervention Technologies (BITs), inspired by SPARX but updated and diversified.

The need for a digital platform was envisaged to connect the e-Screening and the BITs such that positive screening for mild to moderate problems could lead to automated recommendation to try e-Therapy as well as connection to the broader network of existing services (including 24-hour help via YouthLine, https://www.youthline.co.nz/ and first-line face-to-face support such as through guidance counselors and school nurses for secondary school students). The long-term aim was (and still is) to reach high-needs youth, most essentially Māori and Pasifika youth, through deployment in schools in the areas of greatest socio-economic deprivation, in communities and through the Internet with BITs tailored to mobile devices. The need for an underlying digital platform was further consolidated by the research need to study in detail the patterns of usage of the BITs, particularly to determine overall adherence and the most used features for different types of users. Further, to suit different user preferences, the expectation was that a suite of BITs featuring different interaction and intervention styles would be required - i.e. not just one single app. The digital platform would provide a common set of services to support research, and ultimately wider delivery, across this ecosystem of e-Screening and multiple therapeutic BITs.

In addition to the digital platform development work stream, HABITs consists of major work streams in stakeholder engagement and in BITs development. These work streams serve to feed evolving requirements back to the digital platform stream, including data management requirements, and the overall context and nature of acceptable interventions. BITs development has followed a codesign methodology working with secondary schools with strong Māori and Pasifika representation. This co-design process allows the students themselves to participate significantly in providing creative input into apps as well as (in keeping with more traditional user-centered design) providing feedback on designs created by experts. The co-design process as applied to youth e-Health, including an example from HABITs app development, has been reported previously [11].

The initial period of the digital platform work stream (through mid-2019) was modeled as a spiral development process [12] as illustrated in Figure 1. The initial spiral aimed at a technology proof-ofconcept with successive spirals aiming to provide wider capability reaching actual target users at increasing scale. The choice of spiral development 
was based on the knowledge that the digital platform work stream would always be responding to input from the other work streams. Input from the codesign process with youth has included a move to support self-enrolment in the digital eco-system (young people creating their own accounts and providing informed consent to experiments online), elements of the look-and-feel of the portal, and underlying interoperability requirements based on the nature of the BITs emerging from co-design. Further, the digital platform must respond to the research questions emerging from the co-design process and address the resultant series of research protocols.

As HABITs is moving toward a wider evaluation and deployment phase (2019-2024), the overall research methodology aligns with the RE-AIM (Reach, Effectiveness, Adoption, Implementation, and Maintenance) framework [13, 14]. RE-AIM is particularly appropriate given the observed gap between the efficacy of digital mental health tools in clinical trials as compared to their success when subsequently deployed to real-world settings, where uptake and adherence tend to lag [15]. The digital platform development efforts are now working as a component supporting this RE-AIM based approach.
Particular influences of this on platform requirements include: the tracking of representativeness of access, uptake and sustained use of BITs, especially for highneed groups; monitoring that BITs are used with fidelity (e.g. in terms of frequency and persistence of use); and the ability of the solution to be easily maintained for long-term use by participating sites.

As an additional element to the digital platform development approach, from the start the effort was co-funded by the philanthropic organization CureKids with a mandate to support apps for good parenting (as with HABITs, particularly for highest need groups, especially Māori and Pasifika families and those with highest socio-economic deprivation). It was expected that a digital platform infrastructure supporting youth e-Screening and e-Therapy would have many components in common with one to support parents of younger children. As such, the platform was designed from the start to have sufficient flexibility to support both the use-case of making promoting teens wellbeing (through direct use by teens) and the use-case of helping parents to improve their skills and develop stronger families.

In the next three sections we report results of the HABITs digital platform work stream beginning with

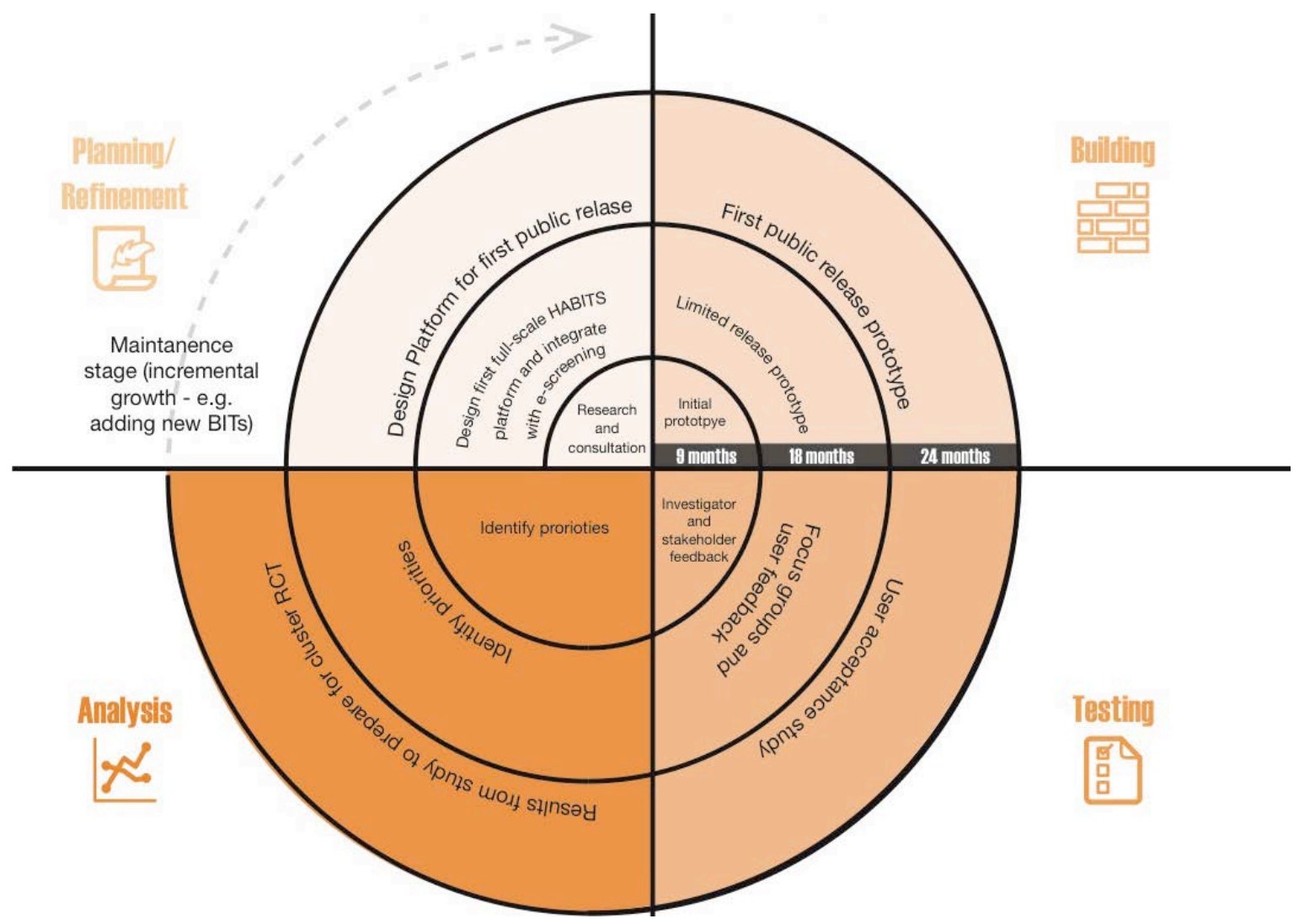

Figure 1. Initial spiral development plan for HABITs digital platform 
initial requirements and proof-of-concept, then looking at learnings as the use of the system has expanded, and lastly looking in more depth at the features created to support research and deployment protocols.

\section{Initial requirements and technical proof of concept}

This phase has been reported previously [16]. Herein we summarize key elements for completeness and with some updates.

The initial proof-of-concept target was to demonstrate the ability to integrate e-Screening and e-Therapy with youth, clinical and trial manager views on a Web portal as appropriate to deployment in a school setting (with the school nurse constituting an example clinical user). Functional and nonfunctional requirements were collected to meet this target in consultation with the broader HABITs investigator team, taking on initial reports from the stakeholder engagement work stream and through research. In addition, personas were developed (validated, and in fact substantially written, by the team members with counseling experience) to further support requirements analysis.

The emerging key functional requirements fit four major categories: (1) identity management; (2) assessment collection and reasoning; (3) usage logging; and (4) workflow support.

Identity management. Important features here include role-based access and minimality of collection and spread of information (e.g. clinical users having access to a degree of identifiable information about youth users at their site, whereas research users may see fine-grained BITs usage data and assessments but only in de-identified form).

Assessment collection and reasoning. This entails the ability to administer mental health assessments to youth users including standardized instruments (e.g. the GAD-7 for anxiety [17]), store data and 'reason' on it (e.g. to interpret a GAD-7 score as 'mild' anxiety and use that as an inclusion criterion for a trial of a specific BIT) and ultimately use scores to assess effectiveness.

Usage logging. This addresses the need to collect fine-grained user-specific details of BITs usage to support research questions about whether apps are being used as intended and how patterns of usage correlate to outcomes.

Workflow support. Initially this requirement was understood as 'consent and randomization' (i.e. to support key steps of a randomized-controlled trial, RCT), but further experience revealed associated requirements such as the ability for youth to create their own accounts for self-enrolment in a trial, and support for a broader set of workflows than simply a two-armed (treatment versus control) individuallyrandomized RCT, as well as the need to support the user in navigating the intended steps of a trial.

In addition to the functional requirements above, a number of non-functional / business requirements were identified. One key business requirement was to provide a high-quality user experience, especially for language, iconography and expectations to be acceptable and welcoming for Māori and Pasifika teens. A further aspect of the business requirements was to create a platform that could scale to nationallevel roll-out (with peak load foreseen as a highpercentage of schools deciding to do e-Screening on the same day) and could be maintained through development, trials and wider deployment (estimated at 10 years).

A technology scan was conducted to find technologies suitable to requirements. Key technologies selected included Firebase identity management [18], Drools rule engine [19] for reasoning on clinical assessments and MongoDB [20] for persistence. Components are integrated via Application Programmer Interfaces (APIs) based on Restful Web Services, with Swagger [21] providing documentation and testing support, and JSON payloads for information exchange between the HABITs platform and end-user applications (e-Screening and e-Therapy BITs) which can be stored relatively directly in MongoDB. Health Level 7 Fast Healthcare Interoperability Resources (HL7 FHIR) [22] were initially identified (and used in the first proof-of-concept prototype) to implement data transfer of questionnaire results (e.g. a completed GAD-7), but were later abandoned in favor of a simpler JSON payload. While attractive due to wide uptake in implementation of health information systems, it was found that our system has minimal overlap with the concepts (and hence FHIR objects and services) of a conventional clinical system.

The resulting architecture is illustrated in Figure 2. The core digital platform itself has been implemented largely in Java Spring Boot [23] with a single Tomcat server but is amendable to crosslanguage development and deployment to multiple servers. Use of a persistence layer compartmentalizes coding that would otherwise tightly couple the business logic layer with the specifics of MongoDB. The Web portal was created using AngularJS. Testing and documenting of a technology proof-ofconcept was completed in June 2017, including Web portal features for admin users to create user accounts and for clinical users to review e-Screening results of youth users. 
Through the latter half of 2017 the platform was refined and interoperability tested with an Android app developed specifically for HABITs that provided 3 modules of CBT based activities. In late 2017 and early 2018 that platform and app were used to conduct two rounds of field trials of the app enrolling a total of 37 students from Auckland area schools. After some initial technical difficulties (chiefly in compatibility of security certificates with older mobile phones - many students having 'hand-me-down'

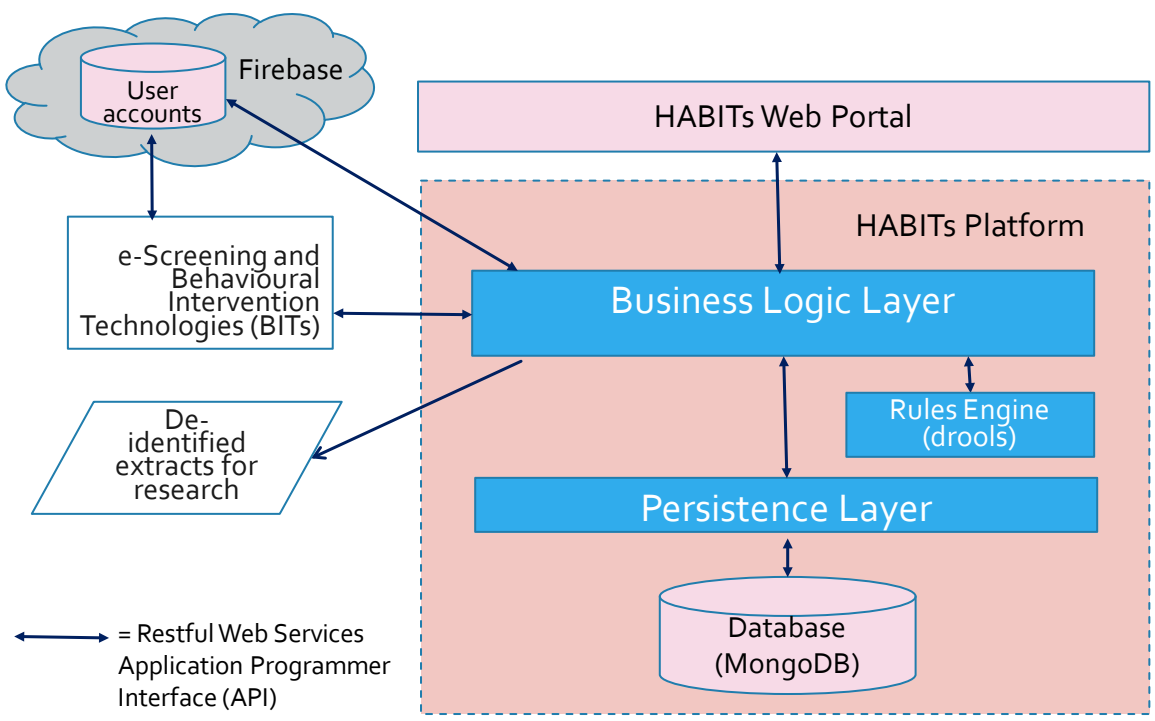
phones from their parents with

Figure 2. HABITs architecture old operating system versions and little free memory), the integrated system performed successfully. Logged usage data was interpretable and combined with student feedback provided guidance for a significant revision of the app (which became the Quest - Te Whitianga app discussed in the next section).

\section{Toward digital mental health ecosystem}

After the initial app field trials, the digital platform effort entered a phase of application to an increasing range of user studies and BITs deployments, which is still ongoing. Key elements of this phase include negotiating technical integration with an increased range of e-Screening tools and apps, supporting the diversity of protocols for 'onboarding' and study of users, and providing distinct user experiences for multiple concurrent trials.

Through this phase we have identified several aspects of interoperability cases: (1) bespoke versus 'commodity' questionnaire tools; (2) modular versus 'single-threaded' apps; (3) native apps versus Web apps; and (4) creation of user accounts from the portal versus apps with their own onboarding and/or identity management. Table 1 summarizes BITs tested with the HABITs platform and key features from an interoperability standpoint. In the remainder of this section we elaborate on lessons learned and capabilities implemented.

Bespoke versus 'Commodity' questionnaire tools. The platform was initially designed to interoperate with the YouthCHAT system, as this system was validated as acceptable with NZ youth. Moreover,

YouthCHAT was developed by collaborating investigators of the team and thus was technically and operationally feasible to adapt to work with the HABITs API. During the 10-year history of eCHAT, however, software solutions for online surveys have become increasingly commonplace. Expertise to configure surveys with these tools is widespread, and they are relatively easy to use. Moreover, research protocols frequently utilize baseline and follow-up assessments, as well as assessments to establish if potential subjects meet study inclusion criteria. Unlike the screening workflow of eCHAT (which for efficiency uses gatekeeping questions in advance of full assessment), the usual approach for study assessments is to ask all the questions of standardized survey instruments as a battery to achieve a score. To exploit the ease of implementing assessments with these commodity survey tools, integration with the HABITs platform was explored. REDCap (https://www.project-redcap.org/) was selected for

Table 1. HABITs platform integration

App Characteristics

Youth

\begin{tabular}{ll}
\hline Quest - & Native app, modular \\
Te Whitianga & \\
HeadStrong & Chatbot, own onboarding \\
Tune In & $\begin{array}{l}\text { Native app, modular, own } \\
\text { onboarding (under development) }\end{array}$
\end{tabular}

Parenting

Play Kindly Native app, single-threaded
Kākano Web app, modular

Super Kids Native app, single-threaded 
integration because it could be run on a local server (maintaining control of confidential data) and had sufficient in-built configurability to allow data exchange with the platform.

Modular versus 'single-threaded' apps. The initial app for integration with the platform was modular in that the app had a main menu to select from a set of independent activities, loosely held together by an overarching storyline. In response to user testing feedback, this app was re-designed. Now called Quest - Te Whitianga, it has been given a new storyline and revised from three to six modules. Remaining consistent for this app was a focus on the younger and less severe end of our audience, a relatively light-hearted motif with gamification, and the modular structure allowing brief in-and-out engagement (it might be termed 'snackification') with CBT and positive psychology based activities. Given this structure, usage logging via the API could be largely satisfied in terms of records of each module session. In Quest - Te Whitianga each session included a brief self-assessment before and after a 'game' that would be played for a duration and may achieve completion of a level.

As we have expanded the ecosystem of apps interoperating with the platform, we have encountered different app workflows dictating different usage logging requirements. The most notable difference is apps that are 'single-threaded' as compared to having a set of modules where the user chooses the order. One single-threaded case is the parenting app Play Kindly which has a series lessons each involving a video regarding a parenting skill and subsequent quiz on knowledge of the skill. To represent usage of this app we record video play events (including re-plays) and quiz-response submission events. A more divergent structure arises with our HeadStrong chatbot. This conversational robot has a small set of relatable young-person characters with avatars, each with their own stories that relate to CBT principles (designed to work with teens who are older and/or have more significant problems than those in the primary group for Question - Te Whitianga). Here the usage logging requirements are more based on events in the dialog flow, notably cases where the user input was matched to a therapeutic response in the chatbot's knowledgebase (e.g. for text indicating interest in suicide they are given empathetic feedback and asked if they wish to link to a $24 x 7$ live human resource).

For each app we have needed to liaise with both the app designers and the researchers to match the research questions to events in the software code. Table 2 summarizes our resultant framework for potential types of usage to log. To support this, the
HABITS platform API for usage logging has a fixed component including event time and the ID of the app (the ID of the user being derived from the Firebase token that authenticates the API access), but also has a flexible attribute-value pair component to tailor the data to the usage event. A particularly challenging type of data to capture technically is the duration of use of an app since the user might not sign off, and might leave the app in the background (or foreground) on their device while doing something else. We have encouraged developers to include a running total time for the app so that we can derive time-per-day or per-week from the event logs, but we expect that event counts (modules completed, videos played etc.) over time will be the more useful indicators of intensity of use.

\section{Table 2. Framework for types of usage data to record in the HABITs digital platform}

$\begin{array}{ll}\text { Event type } & \text { Examples } \\ \text { Access } & \begin{array}{l}\text { Login / opened app } \\ \text { Module launched }\end{array} \\ \text { Engagement } & \text { Video played } \\ \text { markers } & \begin{array}{l}\text { Quiz answers (correct / incorrect) } \\ \text { Chatbot: user input that invoked a } \\ \text { therapeutic response }\end{array} \\ & \begin{array}{l}\text { Levels completed (with time from } \\ \text { start to completion) }\end{array} \\ \text { Achievement } & \begin{array}{l}\text { Prizes unlocked } \\ \text { Ecological momentary } \\ \text { assessments: pre and post }\end{array} \\ \text { Assessment } & \text { Standardized assessments (e.g. } \\ \text { GAD-7) } \\ \text { Module close (time played) } \\ \text { Logout / title menu close (total } \\ \text { time played) }\end{array}$

Native apps versus Web apps. For native apps (such as Quest - Te Whitianga and Play Kindly) the HABITs portal provides a download link and then the user moves out of Web browsing to the native app user experience. Depending on the research protocol, we may recall them to the Web browser later with a message notification containing a link. Conversely, some apps work within the browser (hence 'Web apps'). This is the case with the Käkano parenting app and indeed with our questionnaire tools (both YouthCHAT and REDCap). For continuity, the Web portal can provide a link directly into the Web app, and in the case of questionnaire tools provides a URL for return to the HABITs portal upon completion of the e-Screening or assessment questionnaire. This places an additional interoperability requirement on 
the questionnaire tool to process this return link as well as sending assessment data to the platform. The return navigation provides valuable opportunities such as thanking the user for completing the survey (and possibly indicating next stages in the protocol) or providing feedback and recommendations tailored to their e-Screening / self-assessment findings.

Creation of user accounts from the portal versus apps with their own onboarding and/or identity management solutions. The final technical interoperability case we have needed to accommodate for the ecosystem is where the app already has its own process for establishing the user identity (i.e. setting up a login that is managed by some solution - often Firebase as we have chosen but with another account - and doing initial collection of user profile information). Such an approach is attractive if the app itself is the recruitment pathway - the user discovered the app not the HABITs portal first - and/or if the design of the app includes significant user experiences before suggesting they create an account. Moreover, it can simply be that the app is already engineered around its own identity management solution to such an extent that it is undesirable to re-engineer it around the HABITs platform's approach. To accommodate this we use the Firebase capability to generate custom tokens [24]. Through a process as illustrated in Figure 3, an app that has been issued a HABITs API access key can call the digital platform to access Firebase on the app's behalf to retrieve a custom token. The app then verifies that token against Firebase to gain the access token and subsequently make API calls against our platform as per usual. This coordination only requires some common piece of information about the user, such as an email used with the app and the platform, or could even proceed with HABITs creating a new user account based on enrolment information sent by the app.

Beyond interoperability of the ecosystem components, a significant and ongoing direction for the platform is to support workflow as described in the next section.

\section{Workflow support}

A growing area of the digital platform is the support for workflow. Herein workflow applies broadly to steps around the user experience, including steps of a clinical trial (e.g. giving informed consent), but equally encompasses steps in non-trial use (where we might have account creation, agreement to terms of use and privacy policy, provision of preferred contact details and an initial eScreening ahead of offering access to e-Therapy).
The platform's data model maps users into 'trials' and 'sites' and provides a Web portal experience corresponding to the workflow of that trial, possibly modified by their site (e.g. which school they attend). For some trials there may just be a single site; in other cases call for cluster randomization such that the site dictates which arm of the trial users are in. We use the 'trial' membership concept broadly, including mapping demonstration and test user accounts to demo/test trials, and implementing deployment for routine use as a 'trial' with workflow that constitutes the youth (or parent) user experience.

Our experience has identified a range of opportunities and requirements with respect to workflow. User self-enrolment in trials is proving to be a powerful feature, providing efficiency and other types of superiority over more manual methods. Our primary users (teens and parents of young children) are accustomed to creating their own accounts (e.g. for social media), including providing brief profile information (e.g. contact details by mobile phone or email) and thus find the process acceptable and familiar. This replaces the need for a research assistant to collect and input information by letting the user do it for themselves (and as such under their own direct control). Further, where the user is providing informed consent we have the option to provide an explanatory video (e.g. see Figure 4). There is evidence in the context of consent to clinical procedures that an information video provides better comprehension, and possibly better participation as well as equity in comprehension for those with lower reading ability [25-27].

Our digital platform has a data template for each 'arm' of a trial, with each arm having a different workflow. A so-called open trial has just a single

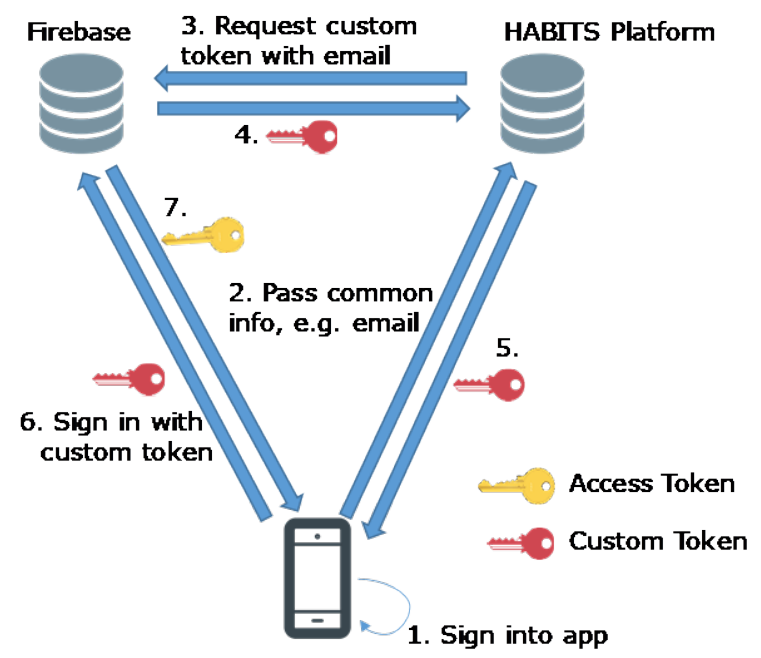

Figure 3. Authentication via custom token 
arm, where a classic RCT has two arms (treatment and control). Our Quest - Te Whitianga RCT is implemented using five arms, although it is a twoarm trial from a statistical perspective, to complete the user experience for those not meeting eligibility for randomization. After consent users complete baseline questions that assess symptoms of anxiety and depression. Those with mild to moderate (but not severe) levels of at least one of these are randomized to the treatment or control arms. Users with minimal symptoms are given brief feedback. Two further arms are for users with severe symptoms or who indicate they are already using mental health services, each receiving different advice (notably links to available services and notification to the trial manager for severe problems and no current mental health service use). All are still invited to download the app if they would like to give it a try.

We specify the logic of each arm as a series of 'status' steps with specified activities and timings. In the control arm of the Quest - Te Whitianga RCT, after meeting inclusion criteria and randomization to the control arm, the next step is for the users to be invited to download a control app (providing some fun facts, but not CBT). After four weeks the next step is invoked and they are sent a link for follow-up assessment. Completion of the survey invokes the next step where they are given the link to the treatment app, and later they are prompted for reassessment and feedback on the app as final steps. Each of these reminders, with configurable text, is automatically sent via the platform's messaging services using the user's indicated preferred contact method. The portal view for trial-manager users allows them to review the status of each participant in their trial.

While the digital platform allows protocols to be substantially specified through input templates, the process is not entirely free of programming. At this stage the user interface of the portal content implementing trial arm steps (as in Figure 4) is via HTML / JavaScript / CSS coding. We are exploring the degree of re-use possible in this coding, but at this stage ensuring sufficiently tailored language and presentation is in part a bespoke Web page development process, although one that uses a suite of common services provided by the platform's business logic layer.

The Platform is currently being used successfully to support trials. As of August 2019, the two largest trials are Kākano with 137 parents of children aged 25 enrolled and Quest - Te Whitianga with 58 young people aged 11-16 enrolled. In addition, there are about 20 platform users in support roles including trial managers, research assistants and test users.

\section{Discussion and conclusion}

In this paper we have presented a digital platform based on Web Services to support research and delivery of digital mental health. This was developed in the context of an ongoing applied research program, called HABITs, to support the wellbeing of New Zealand youth through improved access to e-Screening and e-Therapy tailored to local needs through co-design and iterative field trials. The digital platform provides a suite of reusable services for research and deployment of behavioral intervention technologies. These services include identity management, assessment and usage tracking, and support for protocol workflow. The services are sufficiently broad to support a range of trial designs including open trials, individual randomization and (site based) block randomization.

By providing a set of common Web services and an associated configurable Web portal it is possible for individual trials to be implemented more quickly and to provide better data capture and higher-quality data management. These advantages are realized by the compressing of development time and leveraging of prior development cost inherent in reuse, as well

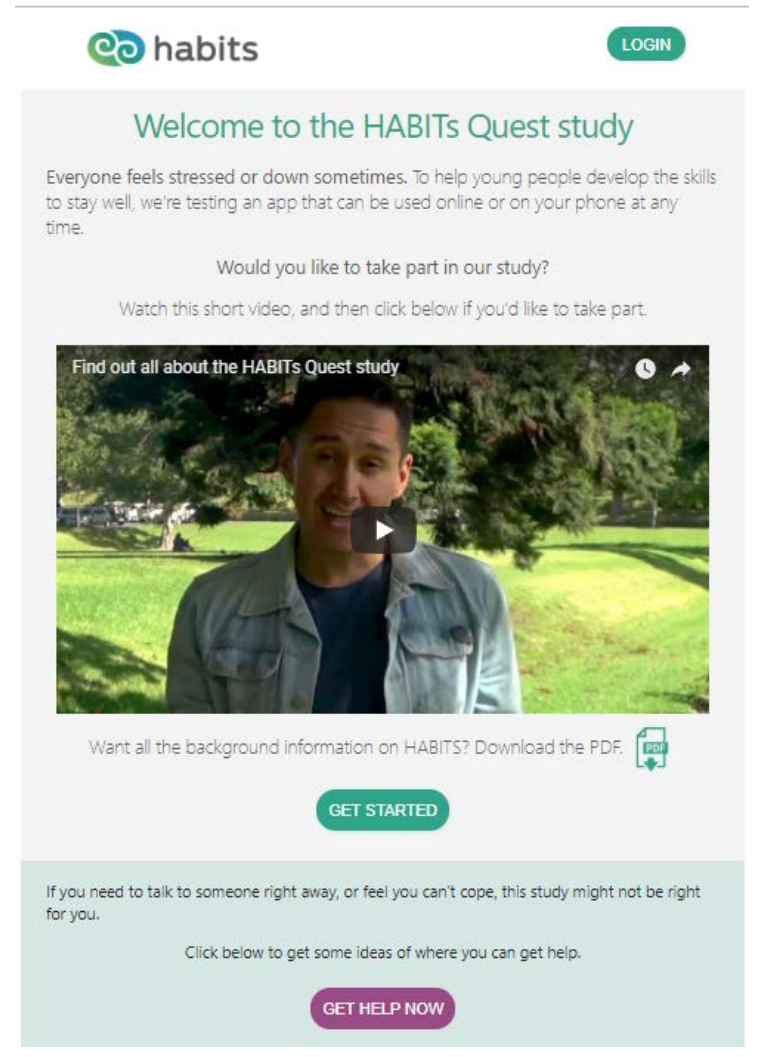

Figure 4. HABITs portal screen for user selfenrolment 
as by benefiting from the prior requirements analysis, technology selection, design and testing that has gone into the implementation of the common components. Additional efficiency is delivered to individual trials through the use of platform/portal services for participant self-registration and automated reminder messages for follow-up assessments, as well as a trial manager portal view to support monitoring and manual follow-up. This infrastructure is being successfully used for a range of apps for youth and parents (as per Table 1), for which it is supporting field trials with numerous self-enrolled participants.

Mohr et al. have identified problems with current digital mental health research, and avenues for solutions [15]. The HABITs program can be seen as a process-level response to their call, and the digital platform as a technology enabler for that response. In particular, Mohr et al. point out that need to fit mental health technologies into their context of use, to design to the users and evaluate in the context of use, as well as to leverage new technologies that can transform mental health delivery. The HABITs program is pursuing such a strategy for NZ youth. The platform facilitates this process through a suite of services, as outlined above, that enables a volume of experimentation with BITs that would be infeasible if each trial required a bespoke trial administration and data management solution. This enhances data capture for successive trials to build on the experience of earlier ones and provides the scalability to move from research trials to sustained deployment of the BITs.

In developing the digital platform we leveraged existing solutions where possible for components of the needed functionality, e.g. Firebase for identity management and REDCap for online assessments. Other technologies could have been incorporated in our architecture and may have reduced development time, enabled additional features and/or resulted in a more robust and standards-based solution. We initially incorporated HL7 interoperability resources, but found the relevant components of limited utility for our needs. An option to consider for our app usage tracking requirements is Google Analytics. In general we see differences between the traffic and return-on-investment focus of Google Analytics and our requirements to understand successful uptake of e-Therapy by specific sub-groups; however, the UserID tracking capability of Universal Analytics [28] shows promise for meeting our requirements and could be an alternative to our current BITs developer API. In addition, we considered whether Informatics for Integrating Biology and the Bedside (i2b2) [29] could form the foundation of our solution. While this would give access to a wide range of data processing tools, the usual i2b2 scenario of integrating existing large-scale electronic medical records with research data seemed remote from the situation with mental health apps. That said, the potential to integrate data from our HABITs platform with broader collections of the Statistics New Zealand Integrated Data Infrastructure (IDI) [30] for long-term follow-up and evaluation is part of our overall research plan.

In conclusion, development of the HABITs digital platform continues alongside further field trials and an increasing focus on deployment of digital mental health solutions for NZ youth. The system has been designed to suit a broader set of requirements around BITs and is supporting trials of apps for parenting skills in addition to the trials and emerging ecosystem of services for teens. We are interested to collaborate with international partners who see potential to utilize the platform services. A larger research and development community would facilitate the sustainability of the software infrastructure, lead to development of new features and broaden the benefits realization.

\section{Acknowledgements}

We thank all members of the HABITs team, and the students, school staff and other stakeholders who took the time to provide input. This work is supported by the New Zealand National Science Challenge 'A Better Start - E Tipu e Rea,' Cure Kids and the Auckland Medical Research Foundation.

\section{References}

[1] World Health Organization, Global accelerated action for the health of adolescents (AA-HA!): guidance to support country implementation, Geneva, 2017.

[2] S. Crengle, T. C. Clark, E. Robinson, P. Bullen, B. Dyson, S. Denny, T. Fleming, S. Fortune, R. Peiris-John, J. Utter, F. Rossen, J. Sheridon, T. Tweevale, and Adolescent Health Research Group, The health and wellbeing of Māori New Zealand secondary school students in 2012. Te Ara Whakapiki Taitamariki: Youth '12, The University of Auckland, Auckland, 2013.

[3] D. D. Ebert, A. C. Zarski, H. Christensen, Y. Stikkelbroek, P. Cuijpers, M. Berking, and H. Riper, "Internet and computer-based cognitive behavioral therapy for anxiety and depression in youth: a meta-analysis of randomized controlled outcome trials.," PLoS ONE vol. 10, no. 3, pp. e0119895., 2015.

[4] Y. Perry, A. L. Calear, A. Mackinnon, P. J. Batterham, J. Licinio, C. King, N. Thomsen, J. Scott, T. Donker, S. Merry, T. Fleming, K. Stasiak, A. Werner-Seidler, and H. Christensen, "Trial for the Prevention of Depression 
(TriPoD) in final-year secondary students: study protocol for a cluster randomised controlled trial," Trials [Electronic Resource], vol. 16, pp. 451, 2015.

[5] H. Christensen, K. M. Griffiths, and L. Farrer, "Adherence in internet interventions for anxiety and depression,” J Med Internet Res, vol. 11, no. 2, pp. e13, Apr 24, 2009.

[6] H. Oinas-Kukkonen, and M. Harjumaa, "Persuasive Systems Design: Key Issues, Process Model, and System Features," Communications of the AIS, vol. 24, no. 28, 2009.

[7] S. M. Kelders, R. N. Kok, H. C. Ossebaard, and J. E. Van Gemert-Pijnen, "Persuasive system design does matter: a systematic review of adherence to web-based interventions,” J Med Internet Res, vol. 14, no. 6, pp. e152, Nov 14, 2012.

[8] S. N. Merry, K. Stasiak, M. Shepherd, C. Frampton, T. Fleming, and M. F. Lucassen, "The effectiveness of SPARX, a computerised self help intervention for adolescents seeking help for depression: randomised controlled non-inferiority trial,” BMJ, vol. 344, pp. e2598, Apr 18, 2012.

[9] F. Goodyear-Smith, J. Warren, M. Bojic, and A. Chong, "eCHAT for lifestyle and mental health screening in primary care,” Ann Fam Med, vol. 11, no. 5, pp. 460-6, Sep-Oct, 2013.

[10] F. Goodyear-Smith, R. Martel, M. Darragh, J. Warren, H. Thabrew, and T. C. Clark, "Screening for risky behaviour and mental health in young people: the YouthCHAT programme,” Public Health Rev, vol. 38, pp. 20, 2017.

[11] H. Thabrew, T. Fleming, S. Hetrick, and S. Merry, "Co-design of eHealth Interventions With Children and Young People,” Front Psychiatry, vol. 9, pp. 481, 2018.

[12] B. Boehm, and W. Hansen, Spiral Development: Experience, Principles, and Refinements, Defense Technical Information Center, 2000.

[13] R. E. Glasgow, T. M. Vogt, and S. M. Boles, "Evaluating the public health impact of health promotion interventions: the RE-AIM framework," Am J Public Health, vol. 89, no. 9, pp. 1322-7, Sep, 1999.

[14] B. Gaglio, J. A. Shoup, and R. E. Glasgow, "The REAIM framework: a systematic review of use over time," Am J Public Health, vol. 103, no. 6, pp. e38-46, Jun, 2013.

[15] D. C. Mohr, K. R. Weingardt, M. Reddy, and S. M. Schueller, "Three Problems With Current Digital Mental Health Research . . . and Three Things We Can Do About Them,” Psychiatr Serv, vol. 68, no. 5, pp. 427-429, May 1, 2017.

[16] J. Warren, E. Tempero, I. Warren, A. Sathianathan, S. Hopkins, M. Shepherd, and S. Merry, "Experience Building
IT Infrastructure for Research with Online Youth Mental Health Tools," in 25th Australasian Software Engineering Conference (ASWEC), Adelaide, 2018, pp. 161-165.

[17] R. L. Spitzer, K. Kroenke, J. B. Williams, and B. Lowe, "A brief measure for assessing generalized anxiety disorder: the GAD-7,” Arch Intern Med, vol. 166, no. 10, pp. 1092-7, May 22, 2006.

[18] Google., Firebase, URL: https://firebase.google.com/, retrieved 6 May, 2018.

[19] Red Hat, Inc., or third-party contributors,, Drools Busines Rules Management System, URL: https://www.drools.org/, 2018.

[20] MongoDB, Inc.,, MongoDB for GIANT Ideas, URL: https://www.mongodb.com/, 2018.

[21] SmartBear Software., World's Most Popular API Framework: Swagger, Somerville, MA; URL: https://swagger.io/: SmartBear Software, 2018.

[22] HL7.org, Overview - FHIR v3.01, URL: https://www.hl7.org/fhir/overview.html, 2017.

[23] Pivotal Software, Inc.,, Spring Boot, URL: https://spring.io/projects/spring-boot, 2018.

[24] Google Developers, Create Custom Tokens, https://firebase.google.com/docs/auth/admin/create-customtokens (last accessed 31 May, 2019), n.d.

[25] J. Weston, M. Hannah, and J. Downes, "Evaluating the benefits of a patient information video during the informed consent process," Patient Educ Couns, vol. 30, no. 3, pp. 239-45, Mar, 1997.

[26] M. J. Rossi, D. Guttmann, M. J. MacLennan, and J. H. Lubowitz, "Video informed consent improves knee arthroscopy patient comprehension,” Arthroscopy, vol. 21, no. 6, pp. 739-43, Jun, 2005.

[27] M. Dunbar, G. Paton, and A. Shinghal, "An Educational Video Improves Consent in Pediatric Lumbar Puncture: A Randomized Control Trial," Pediatric Neurology, vol. In press, 2019.

[28] Google, About Universal Analytics: URL: https://support.google.com/analytics/answer/2790010?hl=e n, retrieved 7 June 2019, 2019.

[29] S. N. Murphy, G. Weber, M. Mendis, V. Gainer, H. C. Chueh, S. Churchill, and I. Kohane, "Serving the enterprise and beyond with informatics for integrating biology and the bedside (i2b2),” J Am Med Inform Assoc, vol. 17, no. 2, pp. 124-30, Mar-Apr, 2010.

[30] B. Milne, J. Atkinson, T. Blakely, H. Day, J. Douwes, S. Gibb, M. Nicholson, N. Shackleton, A. Sporle, and A. Teng, "Data resource profile: The New Zealand Integrated Dat Inframstructure (IDI)," International Journal of Epidemiology, vol. epub ahead of print, pp. 1-6, 2019. 